\title{
Anatomy and physiology of directional hearing
}

\author{
T. Przewoźny \\ Department of Otolaryngology, Medical University of Gdansk, Poland
}

[Received 19 November 2014; Accepted 25 December 2014]

\begin{abstract}
The aim of the paper is to present the most important neuroanatomical and neurophysiological studies concerning the phenomenon of sound localisation in space by people. The author analysed the available literature concerning this topic. The article presents different theories explaining the phenomenon of sound localisation, such as interaural differences in time, interaural differences in sound intensity, interaural and monaural differences in phase and the anatomical and physiological basis of these processes. It also describes methods of measurement of disturbances in sound localisation which are used around the world and in Poland, also by the author of this work. The author lists a number of reports on the neurological causes of disturbances in sound localisation, especially the ones connected with vascular damage of the brain. The reports indicate that it is the temporal and parietal lobes that play the role of cortical centres of sound localisation. Also, it should be pointed out that even though having two ears indeed makes it possible for humans to localise sources of sounds, the process itself takes place in the brain. (Folia Morphol 2015; 74, 1: 9-15)
\end{abstract}

Key words: directional hearing, sound localisation, auditory pathway, spatial discrimination

\section{INTRODUCTION}

The ability of localising sources of sound, also referred to as directional hearing, belongs to auditory abilities of higher level. It is based on identifying the location of sound source in space and being able to recognise the distance between the source of the sound and oneself. The ability to recognise the direction from which sound is coming constitutes one of the main elements of a complex function of the hearing organ, i.e. the ability to localise the source of sound in space. This phylogenetic phenomenon is older than reception of pure tones and speech [12, 15, 31, 40]. In everyday life sound localisation is mainly used for monitoring of the surroundings. Giovanni Battista Venturi (1746-1822), who suggested that localisation of sounds was dependent upon inequalities at the two ears, is considered to be the precursor of studies on sound localisation in humans [45]. Orientation of a human body in a 3-dimensional space requires very complicated processes, which involve the organs of vision and hearing. The centres of tactile sensation localisation are located in the parietal lobe and the centres for object orientation are located in the parietal-occipital area. These observations correlate with the results of the study carried out on blind patients, who were localising sounds as effectively as healthy patients [34]. Scientists have been trying to explain the phenomenon of sound localisation for over 150 years. Below are the most important theories aimed at explaining this phenomenon. 


\section{THEORIES EXPLAINING THE PHENOMENON OF SOUND LOCATION}

\section{Interaural difference in time}

The crossing of the fibres of auditory pathway takes place on several levels: the trapezoid body, lateral lemniscus and between the inferior colliculi of the quadrigeminal lamina. Between these centres takes place a continuous process of inhibiting the side at which the ear was stimulated later by the impulses from the ear that was stimulated earlier [23,30]. Thus, the cochlear nucleus can inhibit the superior olivary nucleus, the superior olivary nucleus inhibits the nucleus of the lateral lemniscus at the opposite side, the nucleus of the lateral lemniscus can inhibit the discharges in the synapses of the inferior colliculi of the quadrigeminal lamina and the colliculus itself inhibits the medial geniculate body of the opposite side [44]. The neuron of the lower layer of the side that was stimulated earlier inhibits the neuron of the higher layer of the opposite side (stimulated later) and does not allow for the inhibition of the side that was stimulated later. This is a system of crossed pathways which inhibit each other (Fig. 1) [26]. Before the sound reaches the more distant ear, there is time delay $(\Delta t)$. Time delay needed for alleged movement of the sound source is $0.018 \mathrm{~ms}[10,21,22,24]$. If the time delay between the stimulation of the right and left ear is between $0.6 \mathrm{~ms}$ and $2 \mathrm{~ms}$, we localise the sound as coming from our side. Time delay longer than 2 ms will be interpreted as two different signals, which is for example used in the Random Gap Detection Test in the diagnostics of central auditory processing disorders (CAPD). The delay of $0.6 \mathrm{~ms}$ is also needed for the flow of inhibiting signal through a synapse. Moreover, inhibition by the non-crossed pathways is observed for centripetal neurons for impulses from the crossed delayed side, blocking their arrival at brain cortex at the side of the earlier stimulus (mainly at the level of the inferior colliculus and the geniculate body). There is also the inhibition of the lower auditory centres by the higher ones in a crossed way. Scientists proved the presence of such fibres in the cochlear nuclei, which partly follow the olivary-cochlear tract $[17,35]$. The theory of time difference mainly pertains to the sound of frequency lower than $1500 \mathrm{~Hz}$, i.e. the lower half of the range of human sensitivity to sounds.

\section{Interaural difference in sound intensity}

The average gauge between the auricles of an adult person is $21 \mathrm{~cm}$. Sound reaches one auricle with a given intensity. Depending on the location of

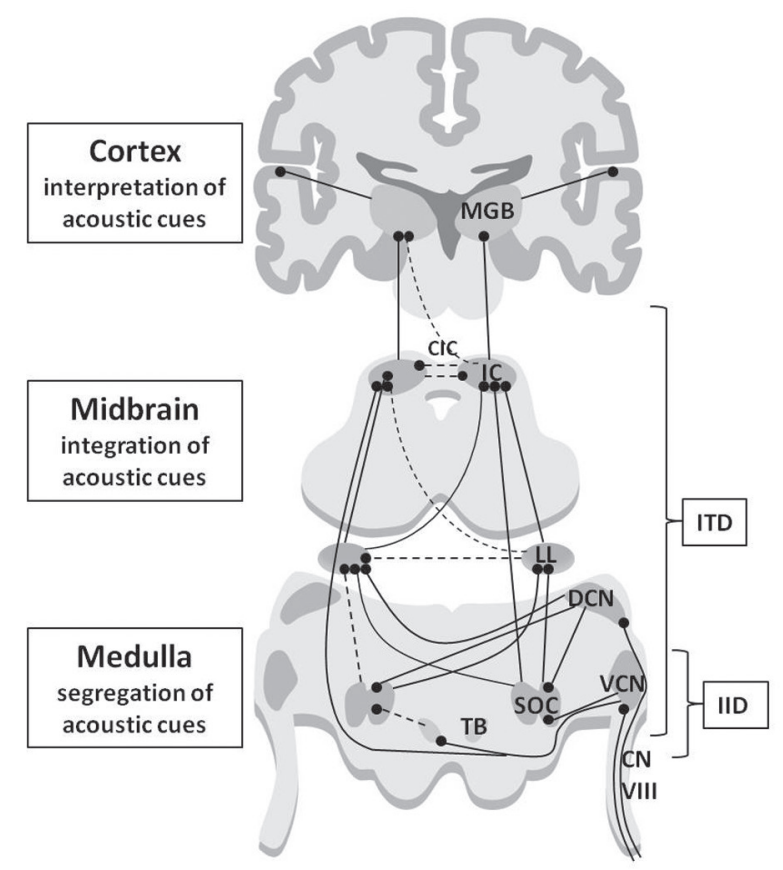

Figure 1. Auditory pathway contributions to directional hearing; CN VIII — cranial nerve VIII (vestibulo-cochlear); VCN — ventral cochlear nucleus; DCN — dorsal cochlear nucleus; SOC — superior olivary complex; TB — trapezoid body; LL — lateral lemniscus; IC - inferior colliculus; MGB — medial geniculate body; CIC — commissure of inferior colliculi; ITD — interaural time difference; IID — interaural intensity difference; black solid line - excitatory fibres; black broken line — inhibitory fibres; black circles - synapses.

the sound source, intensity of the stimulus and head shadow there will be a decrease of sound wave intensity in the ear that is more distant from the sound source. An acoustic wave reaching the head undergoes a process of diffraction and reflection thanks to which the intensity of the wave reaching the ear behind the obstacle and before it will be different. The value of the interaural difference of signal intensity caused by the screening effect of the head and the difference of distance between each of the ears and the source of the sound depends on signal intensity and location of the source of sound in relation to the patient. When the source of sound is positioned centrally to the subject, the quantity of energy reaching each of his ears is the same. It has been observed that this phenomenon plays an especially crucial role in the localisation of sounds heard in horizontal plane [15]. Scientists reported, that the threshold value of the interaural difference of volumes is a function of signal intensity and for the signals of intensity between $70 \mathrm{~dB}$ and $100 \mathrm{~dB}$ over the threshold the lowest noticeable value 
of signal intensity difference reaching both ears is $1 \mathrm{~dB}$. However, von Békésy [47], a winner of the Nobel Prize in medicine in 1961, did not perceive this theory as relevant in explaining sound localisation abilities. The interaural intensity difference-effect occurs for waves of equal or shorter length than the size of the obstacle, that is mainly for frequencies above $1500 \mathrm{~Hz}$, which is the upper half of the range of our sensitivity to sounds.

\section{Binaural and monaural difference in phase}

If two sources of sound are placed at a certain distance from each other in front of one ear and they create a certain angle between them and the auricle, then the spectrum of the sound signal at the auricle will depend on this angle and will be different for the right and left ear. It was observed that the signal is localised at the side which is reached by the leading phase. Along with the increase of phase difference the auditory sensation moves away from the central line and achieves the maximum lateral inclination at the phase difference of $180^{\circ}$ (half of wave length). If phase difference continues to increase, the auditory sensation gradually moves back to the central line, achieving it at the phase difference of $360^{\circ}$ (entire wave length). This proves that the phase difference of $360^{\circ}$ remains undetected by humans. The theory conditioning directional hearing by the difference in phases of the signal stimulating each ear allows for the existence of two mechanisms. The first mechanism comes down to a stimulation of both ears with signals of different phase, which results in asymmetry of stimulation then analysed by the nervous system. The second mechanism presupposes stimulation of each ear with one and the same phase. In this case the nervous system should analyse only the difference in time in which the same signal phase reaches each ear $[7,31,36]$. The theory of phase difference mainly pertains to the sounds of frequency below $1400 \mathrm{~Hz}$, so just as in the case of the theory of time delay, the lower half of the range of our sensitivity to sounds. The phase difference of a signal can be interpreted as a special case of time delays in signals reaching both ears [31]. Additionally this phenomenon is connected with vertical-plane sound localisation [19].

\section{ANATOMICAL BASIS OF THE PHENOMENON OF SOUND LOCATION}

In the light of the theory of this phenomenon, for proper sound localisation people need to have two ears. It has been observed in localisation studies that patients with only one functioning ear showed no ability to discri- minate interaural parameters $[12,46]$. Humans have vestigial muscles moving the auricle (anterior, superior and posterior auricular muscle), which are innervated by the branches of the facial nerve. Only a scarce part of the human population is able to voluntary move the auricles. In was found in clinical conditions that it is possible to electrically stimulate these muscles at the level of brain cortex and that the motor centres are located on the posterior portion of the superior temporal gyrus, close to the end of the Sylvian fissure [52]. The mobility of auricles seems to be significant for sound localisation in mammals, but not in humans. Bats are a perfect example of a use of this phenomenon for hearing [11]. Auricle mobility is associated with the third theory of differences in sound phases. Moreover, the auricle itself is a kind of a filter, mainly for the sounds of frequency higher than $4 \mathrm{kHz}$ [8]. During studies on sound localisation it is important to immobilise the head, because people continuously perform discreet adjusting movements of the auricles, which can distort the results. Animals such as dogs, rodents or bats, continuously perform similar movements to localise objects more effectively, which can be explained with the theories listed above $[2,43]$. The significance of the distal part of the external, middle and inner ear on the phenomenon of sound localisation is not known. It should be pointed out that patients with conductive hearing loss, who suffer from disturbances of tympanic membrane and auditory ossicles present with impairment of sound localisation in all kinds of tests [12]. Interestingly enough, people with bilateral symmetric damage of receptor cells of the organ of Corti are able to properly localise sounds despite hearing impairment. The second neuron of the auditory pathway is constituted by the anterior and posterior cochlear nuclei located at the borderline of the pons and the medulla. Their axons run through the trapezoid body to the lateral lemniscus at both sides, while the number of crossed and non-crossed fibres is more or less the same [3]. They reach the nuclei of the trapezoid corpus, nuclei of the lateral lemniscus, superior nucleus of the oliva and the nucleus of the inferior colliculus of the tectal lamina, whose cells constitute the third neuron of the auditory pathway. The last three mentioned structures constitute the anatomical basis of biaural and sound localisation in the mechanism of interaural time delay, intensity and masking level [9]. Superior olivary complex appears to be predominantly involved in analysing either interaural time or level differences [29]. Thus, pathological processes in this area of the central nervous system significantly impair sound localisation, which has been proven in numerous clinical 
studies of tumours $[12,38,40]$. In the case of tumours of the cerebellopontine angle and focal lesions of the brain stem the disturbances pertain to all studied patients. The fibres come from the nuclei of the brain stem to the nucleus of the medial geniculate body, constituting the fourth neuron of the auditory pathway and the subcortical centre of hearing. Thus, as auditory radiation the neural fibres run through the sublenticular part of the internal capsule to the transverse temporal gyri located in the posterosuperior segment of the superior temporal gyrus (area 41 and 42 according to Brodmann). It is the primary cortical centre of hearing (Al). This area is responsible for general spatial-temporal characteristics of the acoustic information and enhancing sound localisation at the opposite side [39]. Approximately $5-34 \%$ of the neurons of the primary auditory cortex is not stimulated by acoustic stimuli and is probably a reserve. The secondary cortical centre of hearing (All), i.e. the associative auditory centre is located in the lower area of the superior temporal gyrus (area 22 according to Brodmann) [18]. The temporal lobe in humans seems to play a crucial role in the process of sound localisation. Important information about the anatomical basis of this phenomenon was provided by Warren et al. [49, 50] and Maeder et al. [20] in their study with use of functional magnetic resonance. It showed bilateral activation of planum temporale and parieto-temporal operculum during sound localisation. Functional studies show presence of a temporal-parietal pathway for the processing of sound-source motion. Scientists have suggested, that we may be dealing with coexistence of two functionally distinct auditory cortical processing pathways. The first of them - a dorsal 'where' pathway located in the posterior superior temporal plane and preferentially concerned with the processing of auditory spatial information, and the second ventral 'what' pathway originating in the anterior superior temporal plane and preferentially concerned with processing auditory object information, including vocalisations. The results of these studies are consistent with the clinical observations made by other authors $[12,25,33,37,41,48]$. It has also been shown using the most modern technique of magnetoencephalography that a substantial increase in the spatial accuracy of source localisation can result from differing brain geometry in different subjects [16].

Scientists also tried to explain the phenomenon of sound localisation at the neuronal level. It has been discovered that in the cortex of the temporal lobe there are neurons which most probably decode sound localisation throughout $360^{\circ}$ of azimuth based on the spike pattern [27]. The sound localisation behaviour and era anatomy are fine-tuned to the statistics of natural auditory scenes [32].

\section{METHODS OF INVESTIGATING SOUND LOCALISATION}

First attempts to investigate this ability are thought to have been conducted by Politzer, who, in 1867 introduced a method based on identifying the location of sound source by pointing at it with a finger or hand. This method was modified by Preyer in 1881, who asked the subjects to verbally describe the location of the source of sound. In 1901 Pierce suggested that the subject should manually reposition the source of sound to an appropriate location [cited by 42]. Similar method of investigating sound localisation was used in Poland in 1937 by Zakrzewski [53]. In the experiment that he conducted the subject is positioned in the centre of a sphere with 13 loudspeakers and is asked to point with his hand the location of the source of sound and simultaneously describe verbally where the source is. A more advanced method was presented by Matzker in 1957 and 1959 [21-25]. It used an artificial delay of acoustic stimulus reaching both ears. The test was performed in headphones. The reference stimulus was diotic (both ears received the same waveform simultaneously). The smallest change in the parameter that can be discriminated reliably is called just-noticeable-difference in interaural time delay or interaural intensity difference. The first test of sound location in patients with ischaemic stroke was performed by Sanchez-Longo et al. in 1958 [38]. They assessed sound localisation with an earphone of an audiometer placed at 13 different positions at the edge of the ophtalmological perimeter. The angle between the position pointed by the investigated person in relation to the real position of the loudspeaker was the result of his method and the final result was the arithmetic mean value of 13 measurements for different azimuths. Pinek et al. [33] used 6 loudspeakers in a free-field fixed on a semicircular aluminium structure. The signals used were a word spoken and white noise.

The directional hearing acuity angle test (DHAAT) was first described in 1938 by Wilska [51]. She used 2 loudspeakers, which were approached and put in a circle with the patient in the middle. The angle between two radiuses connecting the centre of the circle and points of loudspeakers' positions was the result. DHAAT was first performed in Poland by Zakrzewski [54] in 1960. He used a loudspeaker fixed on a perpendicular, semicircular frame moving horizontally and vertically along a periphery in front of the patient's 
head. He reduced his first device from 1937 with the subject positioned in the middle of a sphere with 13 speakers pointed with hand and simultaneously confirmed orally the position of the sound source to one movable loudspeaker with the subject seated in the middle of the circle. In 1958 Mills [28] described measurement with use of the smallest angular separation that can be detected between the sources of two successive tone pulses, which he called a minimum audible angle (MAA). In this study he indicated that the resolution of the direction of a source is determined, at frequencies below about $1400 \mathrm{~Hz}$, by interaural differences in phase or time, and at higher frequencies by differences in intensity. A similar method of measuring directional hearing was used by Häusler et al. [12, 13] and Elfner et al. [5], who enriched the set of tests by introducing a horizontal MMA at 8 reference azimuths around the subject's head. The method proposed by Mills and Häusler is successfully used (with small alterations) by the author of this article for diagnostic evaluation of CAPD in the Clinic of Audiology of the University Clinical Centre. Figure 2 presents a fragment of the MAA study.

In the method used by the author of this article the MAA test is conducted in a soundproof booth. Signals are generated by using noise bursts $80-12.500 \mathrm{~Hz}$ presented in sets of two 1-s bursts, with acoustic pressure level of $85 \mathrm{~dB}$ sound pressure level. Signals are amplified and transmitted to the loudspeaker set. The measurement is made in the free-field in the horizontal plane at 8 equally spaced angles (every $45^{\circ}$ ) around the head for azimuths: $0^{\circ}, 45^{\circ}, 90^{\circ}, 135^{\circ}, 180^{\circ}, 225^{\circ}, 270^{\circ}$, $315^{\circ}$ and each time calculating average value of angle.

\section{A CLINICAL STUDY OF DISTURBANCES IN THE FUNCTION OF SOUND LOCALISATION CENTRES}

Types of hearing impairment in humans, resulting exclusively from brain damage are diverse. The possibilities were described by Earnest et al. [4]. Unilateral changes in the cortex of the temporal lobe result in mild hearing impairment. The impairment can be caused by a difficulty in discrimination of the distorted, slowed or quickened speech stimulating the other ear [14] or a disturbance of sound localisation in the opposite auditory field [38]. Ipsilateral damage of the temporal lobe in the dominant brain hemisphere (in right-handed people it is the left hemisphere), most frequently causing hearing loss, may not affect sound localisation [6]. Being deaf to pure tones is much more frequently a result of bi-

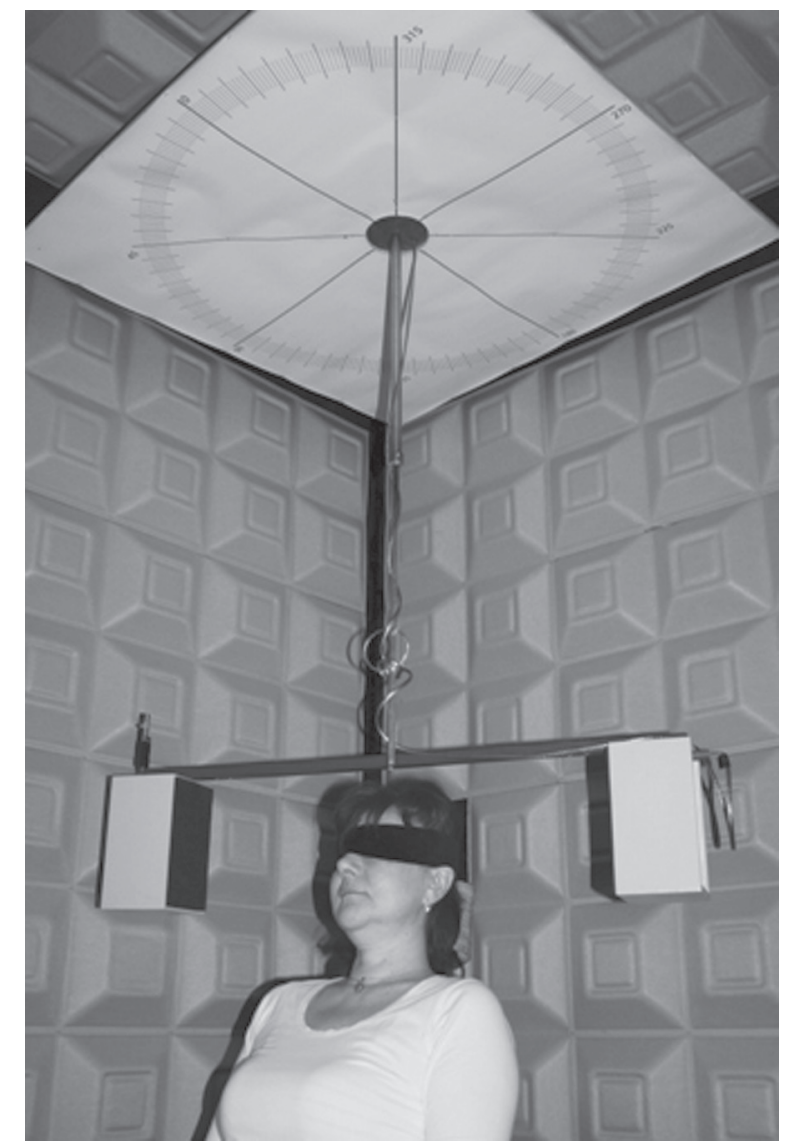

Figure 2. Horizontal minimum audible angle (HMAA) study in a 51-year-old woman. View from sound-proof booth.

lateral lesions in the superior temporal cortex [8]. Many investigators assessed sound localisation disturbances in patients with brain tissue damage $[1,12,33,38,55]$. Sanchez-Longo et al. [38] examined 50 patients, out of whom 10 were after cerebral stroke. Sound localisation disturbances were revealed in 7 per every 10 patients (70\%). Among 21 subjects with pathological involvement of the temporal lobe, 19 had sound localisation disturbances, mainly at the opposite side. Similar clinical observations of patients with vascular brain damage were made by the author of this article (Fig. 3).

Pinek et al. [33] observed unimodal spatial auditory deficits in patients with left posterior lesions and milder localisation deficits in patients with extensive parietal damages what contributed thesis of dissociation between localisation and other auditory processing. Bisiach et al. [1] investigated a group of 107 patients with vascular ssbrain changes and found that a defect of spatial hearing involved both contralateral and ipsilateral hemispheres to the brain lesions. Other clinical studies indicated that sound localisation is even more impaired by a phenome- 


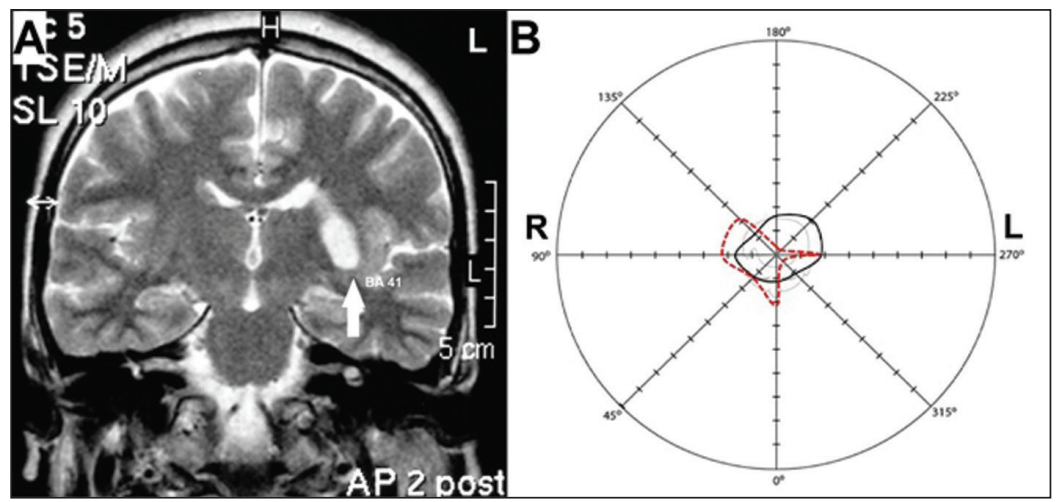

Figure 3. A. Result of magnetic resonance imaging examination, frontal section through the forebrain at the level of the mammillothalamic tract in a 31-year-old patient with ischaemic stroke of the left brain hemisphere and normal hearing. The arrow points the ischaemic focus in the area of the posterior branch of the internal capsule, putamen and pale globe in the direct proximity of the insula and the parieto-temporal operculum; B. Result of a test of sound localisation on day 7 after a stroke. Obvious disturbance of sound localisation at the right side (azimuth $90^{\circ}, 135^{\circ}$ ) and at the front (azimuth $0^{\circ}$ ), opposite to the stroke focus; black solid line — norm range; red broken line — test result.

non of spatial neglect occurring in right superior temporal cortex damage [55]. As shown in clinical studies, sound localisation is determined by the elements of the centripetal auditory pathway and its centres located in the cortex of the temporal lobe, at the borderline with the parietal lobe. The presence of sound localisation disturbances in the case of lesions located in lobes other than the temporal and parietal one is difficult to explain. Some researchers try to interpret it as a compression of the involved lobe on the temporal one or as disturbances in the cerebrospinal fluid flow [38].

\section{CONCLUSIONS}

Sound localisation takes a new scientific meaning in the light of the Nobel Prize in physiology or medicine awarded in 2014. May-Britt and Edvard Moserow with John O'Keefe discovered groups of nerve cells, which coordinate the system responsible for precise orientation in space, an "inner GPS" that makes it possible for people to orient themselves in space. It is made of by grid cells, which form networks in the hippocampus. Sound localisation and its anatomical and neurophysiological basis have been an object of scientists' interest for over 100 years. Acquisition of knowledge on this topic is visible in literature and is certainly not finished yet. Further studies will for sure finally explain all the complex neurophysiological processes behind proper localisation of sounds in space.

\section{REFERENCES}

1. Bisiach E, Cornacchia L, Sterzi R, Vallar G (1984) Disorders of perceived auditory lateralization after lesions of the right hemisphere. Brain, 107: 37-52. doi: http://dx.doi.org/10.1093/ brain/107.1.37.
2. Brown CH, May BJ (2005) Comparative mammalian sound localization. In: Popperand AN, Fay RR ed. Sound source localization springer handbook of auditory research. Springer, New York, pp. 124-178.

3. De Myer W (2003) The vestibulocochlear system. In: De Myer W ed. Neuroanatomy. 2nd Ed. Williams \& Wilkins, Baltimore, p. 214.

4. Earnest MP, Monero PA, Parnell PR (1977) Cortical deafness. Demonstration of the pathologic anatomy by CT scan, Neurology, 27: 1172-1175.

5. Elfner LF, Howse WR (1987) Auditory localization in a free field using discrimination procedures. J Aud Res, 27: 1-14.

6. Gazzaniga MS, Glass AV, Sarno MT, Posner JB (1973) Pure word deafness and hemispheric dynamics: a case history. Cortex, 9: 136-143.

7. Getzmann S (2004) Spatial discrimination of sound sources In the horizontal plane following an adapter sound. Hear Res, 191: 14-20.

8. Goldstein MN, Brown M, Hollander J (1975) Auditory agnosia and cortical deafness. Analysis of a case with three year follow-up. Brain Lang, 2: 324-332.

9. Grothe B, Pecka M (2014) The natural history of sound localization in mammals: a story of neuronal inhibition, Front Neural Circuits. 8 (116): 1-19. doi: 10.3389/fncir.2014.00116.

10. Hancock KE, Delgutte $B$ (2004). A physiologically based model of interaural time difference discrimination. J. Neurosci, 24: 7110-7117. doi: 10.1523/JNEUROSCI.0762-04.2004.

11. Harnischfeger G, Neuweiler G, Schlegel P (1985). Interaural time and intensity codingin superior olivary complex and interior colliculus of the echolocating bat Molossusater. J Neurophysiol, 53: 89-109.

12. Häusler R, Colburn S, Marr E (1983) Sound localization in subjects with impaired hearing. Spatial-discrimination and interaural-discrimination tests. Acta Otolaryngol Suppl, 400: 1-62.

13. Häusler R, Levine RA (2000) Auditory dysfunction in stroke. Acta Otolaryngol, 120: 689-703.

14. Jerger J (1960) Observations on auditory behaviour in lesions of the central auditory pathways. Arch Otolaryngol, 71: 797-806. 
15. Jongkees LB, Groen JJ (1946) On directional hearing. J Laryngol Otol, 1946; 61: 494-504.

16. Larson E, Maddox RK, Lee AK (2014) Improving spatial localization in MEG inverse imaging by leveraging intersubject anatomical differences. Front Neurosci, 8 (330): 1-11. doi: 10.3389/fnins.2014.00330.

17. Lorente de No (1933) Anatomy of the eighth nerve. III. General plan of structure of the primary cochlear nuclei. Laryngoscope, 43: 327-350.

18. Luxon LM (1981) The anatomy and pathology of the central auditory pathways. Br J Audiol, 15: 31-40.

19. Macpherson EA, Sabin AT (2013) Vertical-plane sound localization with distorted spectral cues. Hear Res, 306: 76-92. doi: 10.1016/j.heares.2013.09.007.

20. Maeder PP, Meuli RA, Adriani M, Bellmann A, Fornari E, Thiran JP, Pittet A, Clarke S (2001) Distinct pathways involved in sound recognition and localization: a human fMRI study. Neuroimage, 14: 802-816. doi: 10.1006/nimg.2001.0888.

21. Matzker J (1957) The binaural test in space-occupying endocranial processes; a new method of otological diagnosis of brain disease. [Article in German]. Z Laryngol Rhinol Otol, 36: 177-189.

22. Matzker J (1958) Attempted explanation of aural determination of direction on the basis of super-sensitive recording of time differences. [Article in German]. Acta Otolaryngol, 49: 483-494.

23. Matzker J (1958) Model of synaptic connection in the brain stem nuclei of the auditory tract. [Article in German]. Z Laryngol Rhinol Otol, 37: 167-173.

24. Matzker J, Springborn E (1958) Directional hearing and age, Z Laryngol Rhinol Otol, 37: 739-745.

25. Matzker J, Welker H (1959) Testing of directional hearing for demonstration of and topical diagnosis of brain diseases. [Article in German]. Z Laryngol Rhinol Otol, 38: 277-294.

26. May BJ, Anderson M, Roos M (2008) The role of broadband inhibition in the rate representation of spectral cues for sound localization in the inferior colliculus. Hear Res, 238: 77-93. doi: 10.1016/j.heares.2008.01.008

27. Middlebrooks JC, Clock AE, Xu L, Green DM (1994) A panoramic code for sound location by cortical neurons. Science, 264: 842-844.

28. Mills AW (1958) On the minimum audible angle. J Acoust Soc Am, 30: 237-246.

29. Moore JK, Osen KK (1991) Anatomy and physiology of binaural hearing. Audiology, 30: 125-134.

30. Neff WD (1957) Behavioral studies of auditory discrimination. Ann Otol Rhinol Laryngol, 66: 506-513.

31. Nordlund B (1962) Physical factors in angular localization Acta Otolaryngol, 54: 75-93.

32. Parise CV, Knorre K, Ernst MO (2014) Natural auditory scene statistics shapes human spatial hearing. Proc Natl Acad Sci USA, 111: 6104-6108. doi: 10.1073/pnas.1322705111.

33. Pinek B, Duhamel JR, Cavé C, Brouchon M (1989) Audio-spatial deficits in humans: differential effects associated with left versus right hemisphere parietal damage. Cortex, 25: 175-186.

34. Pruszewicz A, Gerwel T (1964) Studies on the auditory localization in blind persons (measurement of the angle of acuity of directional hearing in a free auditory field). [Article in Polish]. Otolaryngol Pol, 18: 231-234.

35. Rasmussen GL (1946) The olivary peduncle and other fiber projections of the superior olivary complex. J Comp Neurol, 84: 141-219.
36. Rayleigh L (1907) On our perceptron of sound direction. Philos Mag, 13: 232.

37. Ruff RM, Hersh NA, Pribram KH (1981) Auditory spatial deficits in the personal and extrapersonal frames of reference due to cortical lesions. Neuropsychologia, 19: 435-443. doi: http:// dx.doi.org/10.1016/0028-3932(81)90073-7.

38. Sanchez-Longo LP, Forster FM (1958) Clinical significance of impairment of sound localization. Neurology, 8: 119-125.

39. Schreiner CE (1991) Functional topographies in the primary auditory cortex of the cat. Acta Otolaryngol Stockh Supl, 491: 7-15.

40. Sonoda S, Mori M, Goishi A (2001) Pattern of localisation error in patients with stroke to sound processed by a binaural sound space processor. J Neurol Neurosurg Psychiatry, 70: 43-49.

41. Szikszay I, Glonning K (1969) Sound localization in various cerebral lesions. Pract Otorhinolaryngol, 31: 212-217.

42. Szmeja Z. (2003) Sound localization. [in Polish] In: Pruszewicz A ed. An outline of clinical audiology. WAM, Poznań, pp. 213-223.

43. Tollin DJ, McClaine EM, Yin TC (2010) Short-latency, goal-directed movements of the pinnae to sounds that produce auditory spatial illusions. J Neurophysiol, 103: 446-457. doi: 10.1152/jn.00793.2009.

44. van der Heijden M, Lorteije JA, Plauška A, Roberts MT, Golding NL, Borst JG (2013) Directional hearing by linear summation of binaural inputs at the medial superior olive. Neuron, 78 : 936-948. doi: 10.1016/j.neuron.2013.04.028.

45. Venturi JB (1796) Considérations sur la connaissance de l'étendue que nous donne le sens de l'ouïe. [Article in French]. Magasin Encyclopédique, ou Journal des Sciences, des Lettres et des Arts, 3: 29-37.

46. Viehweg R, Campbell RA (1960) Localization difficulty in monaurally impaired listeners. Ann Otol Rhinol Laryngol, 69: 622-634

47. von Békésy G (1963) Hearing theories and complex sounds. J Acoust Soc Am, 35: 588-601.

48. Walsh EG (1957) An investigation of sound localization in patients with neurological abnormalities. Brain, 80: 222-250. doi: http://dx.doi.org/10.1093/brain/80.2.222.

49. Warren JD, Zielinski BA, Green GG, Rauschecker JP, Griffiths TD (2002) Perception of sound-source motion by the human brain. Neuron, 34: 139-148.

50. Warren JE, Wise RJ, Warren JD (2005) Sounds do-able: auditory-motor transformations and the posterior temporal plane. Trends Neurosci, 28: 636-643. doi: http://dx.doi.org/10.1016/j. tins.2005.09.010.

51. Wilska A (1938) Untersuchungen über das rihtungshören. [Article in German]. Acta Societ Med Funical „Duodecim”, 21: 1-86.

52. Yu L, Terada K, Usui N, Usui K, Baba K, Inoue Y (2010) Ear movement induced by electrical cortical stimulation. Epilepsy Behav, 18: 488-490. doi: 10.1016/j.yebeh.2010.05.016.

53. Zakrzewski A (1937) From the investigations on diotic sound localization. [Article in Polish]. Pol. Przegl. Otolaryngol, 13: 306-320.

54. Zakrzewski A (1960) Clinical test for the acuity of directional hearing, Bulletin de la Societè des Amis des Science et des Letters de Poznań, Serie C, 10: 7-14.

55. Zimmer U, Lewald J, Karnath HO (2003) Disturbed sound lateralization in patients with spatial neglect. J Cogn Neurosci, 15: 694-703. 